\title{
Brain Injury Patterns in Hypoglycemia in Neonatal Encephalopathy
}

\author{
D.S.T. Wong, K.J. Poskitt, V. Chau, S.P. Miller, E. Roland, A. Hill, and E.W.Y. Tam
}

\begin{abstract}
BACKGROUND AND PURPOSE: Low glucose values are often seen in term infants with NE, including HIE, yet the contribution of hypoglycemia to the pattern of neurologic injury remains unclear. We hypothesized that MR features of neonatal hypoglycemia could be detected, superimposed on the predominant HIE injury pattern.

MATERIALS AND METHODS: Term neonates $(n=179)$ with NE were prospectively imaged with day-3 MR studies and had glucose data available for review. The predominant imaging pattern of HIE was recorded as watershed, basal ganglia, total, focal-multifocal, or no injury. Radiologic hypoglycemia was diagnosed on the basis of selective edema in the posterior white matter, pulvinar, and anterior medial thalamic nuclei. Clinical charts were reviewed for evidence of $\mathrm{NE}, \mathrm{HIE}$, and hypoglycemia ( $<46 \mathrm{mg} / \mathrm{dL}$ ).

RESULTS: The predominant pattern of HIE injury imaged included 17 watershed, 25 basal ganglia, 10 total, 42 focal-multifocal, and 85 cases of no injury. A radiologic diagnosis of hypoglycemia was made in 34 cases. Compared with laboratory-confirmed hypoglycemia, MR findings had a positive predictive value of $82 \%$ and negative predictive value of $78 \%$. Sixty (34\%) neonates had clinical hypoglycemia before MR imaging. Adjusting for 5-minute Apgar scores and umbilical artery $\mathrm{pH}$ with logistic regression, clinical hypoglycemia was associated with a 17.6-fold higher odds of MR imaging identification $(P<.001)$. Selective posterior white matter and pulvinar edema were most predictive of clinical hypoglycemia, and no injury $(36 \%)$ or a watershed $(32 \%)$ pattern of injury was seen more often in severe hypoglycemia.
\end{abstract}

CONCLUSIONS: In term infants with NE and hypoglycemia, specific imaging features for both hypoglycemia and hypoxia-ischemia can be identified.

ABBREVIATIONS: $\mathrm{HIE}=$ hypoxic-ischemic encephalopathy; IQR = interquartile range; NE = neonatal encephalopathy

$\mathbf{N}$ eonatal encephalopathy is a general term used to describe a clinically defined syndrome of disturbed neurologic function in the earliest days of life in the term infant, occurring in 1 to 6 per 1000 live term births, and results in significant mortality and morbidity. ${ }^{1-3} \mathrm{NE}$ may be the result of many etiologies, some of which are treatable (eg, hypoglycemia ${ }^{1}$ ), and others less treatable, such as cerebral dysgenesis or congenital infection. ${ }^{1}$ The most common cause of NE is hypoxia-ischemia, which results in a clinical

Received September 7, 2012; accepted after revision October 6, 2012.

From the Department of Pediatrics (D.S.T.W., V.C., E.R., A.H.) and Department of Radiology (K.J.P.), University of British Columbia, Vancouver, Canada; and Department of Paediatrics, Hospital for Sick Children, Toronto, Canada (S.P.M., E.W.Y.T.).

This work was supported in part by SickKids Foundation and IHDCYH-CIHR National Grants Program.

Paper previously presented in part at: Pediatric Academic Societies Annual Meeting, Boston, Massachusetts, April 28-May 1, 2012.

Please address correspondence to Emily W.Y. Tam, MDCM, MAS, FRCPC, Hospital for Sick Children, Department of Paediatrics, 555 University Ave, Room 6535,

Toronto, ON, Canada M5G 1X8; e-mail: emily.tam@utoronto.edu

- Indicates open access to non-subscribers at www.ajnr.org

http://dx.doi.org/10.3174/ajnr.A3423 constellation of findings termed hypoxic-ischemic encephalopathy. Neonates with NE caused by HIE are at increased risk of depleting their energy stores and developing concurrent hypoglycemia. ${ }^{4}$

When caring for infants with NE, is it important to identify the etiology and time of the brain injury and to predict neurodevelopmental outcome. ${ }^{5,6}$ Neuroimaging with MR has been shown to be the ideal tool to demonstrate the extent of injury and predict outcome. ${ }^{5,7}$ Barkovich et $\mathrm{al}^{8}$ has published the most widely used and validated MR scoring system to diagnose and rate the severity of injury secondary to HIE. In addition to having a normal appearance, infants with suspected HIE may have 4 predominant patterns of injury: watershed, basal ganglia, total, or focal-multifocal injury. ${ }^{7}$

Neonatal hypoglycemia ( $<46 \mathrm{mg} / \mathrm{dL}$ ) occurs in $5 \%$ to $15 \%$ of normal term neonates and can cause visual impairment, epilepsy, and cognitive deficits. ${ }^{9-11}$ Numerous animal and human studies have suggested that neonatal hypoglycemia in the context of HIE may be more detrimental than either condition alone. ${ }^{12-14}$ These studies also suggest that lower glucose levels are associated with a higher severity of encephalopathy and a worse outcome at 2 
years. ${ }^{4,14}$ Neuroimaging studies in neonatal hypoglycemia have shown a correlation between hypoglycemia, parieto-occipital injury, and involvement of the underlying white matter tracts, corpus callosum, and thalamus. ${ }^{8,15,16}$ However, it is unclear whether the pattern of injury seen in HIE is modified by concurrent hypoglycemia and whether the effects of hypoglycemia in the context of HIE can be reliably recognized by imaging alone. The objective of this study was to identify the predominant imaging patterns and specific radiologic features for neonatal hypoglycemia in the context of NE.

\section{MATERIALS AND METHODS Study Subjects}

The study was approved by the institutional Clinical Research Ethics Board and was carried out at a provincial tertiary-level neonatal center. The study cohort consisted of all term infants (36-42 weeks' gestation) with clinically suspected HIE who were seen between July 1, 2004, and December 31, 2010. Standard of care included MR imaging on day 3 of life ( $72 \pm 12$ hours) for all patients with NE and suspected HIE. The timing of imaging has been demonstrated to accurately predict injury on later neuroimaging $^{7}$ and is within the time window for detection of hypoglycemia-associated injury. ${ }^{15}$ Inclusion criteria for this study included term gestational age, NE, a day-3 MR imaging study, and recorded laboratory glucose values obtained at least 12 hours before the MR.

A patient was diagnosed with HIE if at least 1 of the following was present: 1) fetal distress at delivery, 2) Apgar score of $\leq 5$ at 5 minutes, 3 ) requirement for resuscitation at birth, or 4) metabolic acidosis (umbilical artery $\mathrm{pH}<7.1$ or base excess $>10$ ). Charts were also reviewed for date and time of birth and subsequent glucose values. All glucose measurements were counted up to 12 hours before the MR imaging study. Hypoglycemia was defined as $<46 \mathrm{mg} / \mathrm{dL}$ and severe hypoglycemia as $<27 \mathrm{mg} / \mathrm{dL}$. All glucose values and time of measurement were recorded to determine 1) the total number of glucose measurements, 2) the lowest glucose measurement and time of measurement in each subject, 3) the number of normal glucose measurements, 4) the number of low glucose measurements, and 5) the number of separate hypoglycemic episodes.

\section{MR Imaging Studies}

MR imaging was performed according to the methods outlined by Chau et $\mathrm{al}^{7}$ and was performed with sedation on a 1.5T Avanto scanner with the use of VB 16 software (Siemens, Erlangen, Germany). The following sequences were obtained (TR/TE/averages/ field of view/thickness/gap): axial and coronal T1-weighted spinecho images $(800 / 20 / 1 / 230 / 4 \mathrm{~mm} / 0.2 \mathrm{~mm})$, axial fast spin-echo T2-weighted images $(4000 / 101 / 2 / 230 / 4 \mathrm{~mm} / 0.5 \mathrm{~mm})$, and isotropic DWI $b=700,1000(3300 / 82 / 4 / 210 / 4 \mathrm{~mm} / 0.2 \mathrm{~mm})$. Both DWI images and ADC maps were produced. Single-voxel spectroscopy was also performed in the thalami and white matter but was not used in the analysis of the present study.

MR images were reviewed by a single pediatric neuroradiologist blinded to the clinical history and glucose values. The T1- and T2-weighted images, DWI, and ADC maps were reviewed and scored in accordance with Barkovich et $\mathrm{al}^{17}$ and modified by Chau et al. ${ }^{7}$ The extent of injury on each sequence was scored from 0 to 4 in the deep gray matter and from 0 to 5 in the watershed region. The maximal score was used to assign the predominant pattern of injury; watershed, basal ganglia, total, focal-multifocal, or no injury. ${ }^{7}$ All additional MR studies (ie, day-10 or 2-year follow-up scans) were reviewed to validate the assigned pattern of injury.

Concurrent with the MR image review, cases of suspected imaging-positive hypoglycemia were identified on the basis of a constellation of observations. Criteria included bilateral, symmetric selective white matter edema, a posterior predominance to white matter edema, associated edema in the pulvinar and anterior medial thalamic nuclei, and restricted diffusion in the optic radiations. Although the identification of suspected hypoglycemic cases was a subjective decision by the pediatric neuroradiologist, subsequently each imaging feature suspected to be associated with hypoglycemia was individually scored as present or absent.

\section{Data Analysis}

Statistical analysis was performed with Stata 11 software (Stata Corporation, College Station, Texas). Descriptive statistics were used to characterize the cohort for hypoglycemic and hypoxicischemic brain injury. To investigate the association between hypoglycemia and its identification on MR imaging, logistic regression analyses were preformed, adjusting for 5-minute Apgar scores and umbilical artery $\mathrm{pH}$.

\section{RESULTS}

\section{Study Subjects}

Between 2004 and 2010, 192 neonates with NE were identified and were imaged $72 \pm 12$ hours after birth (mean $=78$ hours) without evidence of a cerebral malformation. Of these, 179 (93\%) had glucose measurements performed at least 12 hours before MR imaging. Of the 179 subjects forming the study group, 160 (89\%) had at least 1 clinical indicator of HIE, whereas in 19 subjects, the eligibility criteria were either not recorded or met. The clinical demographics of the cohort are summarized in Table 1. Of note, infants with hypoglycemia had a smaller head circumference at birth $(P=.006)$ and a trend to smaller birth weight $(P=.05)$ and length $(P=.09)$. On the basis of their 5-minute Apgar score or umbilical artery $\mathrm{pH}$, hypoglycemic infants did not show differences in perinatal depression.

\section{Clinical Hypoglycemia}

Glucose measurements were performed at the discretion of the clinical team, and $>1500$ measurements were recorded in our cohort and more than 8 glucose values per subject. In infants without hypoglycemia, there was a median of 5 glucose measurements (IQR, 3-9), and the lowest recorded value occurred an average of 20 hours after birth, or more than 48 hours before MR imaging. Infants with clinical hypoglycemia were tested a median of 11 times (IQR, 7-16), more often than infants without hypoglycemia $(P<.001)$. The mean lowest glucose level was $29 \pm 12$ $\mathrm{mg} / \mathrm{dL}$ and was recorded at a median of 10.25 hours after birth (IQR, 3-33). As outlined in Table 2, hypoglycemia was detected in $60(34 \%)$ neonates. Subjects with HIE included 56 (35\%) with hypoglycemia, of which 18 (11\%) had severe hypoglycemia $(<27$ 


\begin{tabular}{|c|c|c|c|}
\hline & $\begin{array}{c}\text { No Hypoglycemia } \\
(n=119)\end{array}$ & $\begin{array}{l}\text { Hypoglycemia } \\
\quad(n=60)\end{array}$ & $P$ Value \\
\hline Gestational age at birth, weeks, mean \pm SD & $39.26 \pm 1.68$ & $39.12 \pm 1.44$ & .57 \\
\hline Male sex, $n(\%)$ & $64(54 \%)$ & $40(67 \%)$ & .10 \\
\hline Birth weight, grams, mean \pm SD & $3389 \pm 565$ & $3194 \pm 728$ & .053 \\
\hline Birth head circumference, $\mathrm{cm}$, mean $\pm S D$ & $34.90 \pm 2.47$ & $33.80 \pm 1.98$ & .006 \\
\hline Birth length, $\mathrm{cm}$, mean $\pm \mathrm{SD}$ & $51.37 \pm 3.59$ & $50.30 \pm 3.42$ & .090 \\
\hline Umbilical artery $\mathrm{pH}$, mean $\pm \mathrm{SD}$ & $7.05 \pm 0.20$ & $7.02 \pm 0.17$ & .42 \\
\hline Apgar score at 5 minutes, median (IQR) & $5.5(3-7)$ & $6(4-7)$ & .85 \\
\hline Age at MRI, hours, mean \pm SD & $78.06 \pm 15.09$ & $77.03 \pm 16.21$ & .68 \\
\hline
\end{tabular}

Medians are compared by means of the K-sample equality of medians test. Means are compared by means of the $t$ test. Proportions are compared by means of the Fisher exact test.

Table 2: Number of subjects with HIE injury patterns in hypoglycemia in the presence or absence of clinical evidence for HIE

\begin{tabular}{|c|c|c|c|c|c|}
\hline & No Injury & Watershed & Basal Ganglia & Total & Multifocal \\
\hline All subjects, $n=179$ & $85(47 \%)$ & $17(9 \%)$ & $25(14 \%)$ & $10(6 \%)$ & $42(23 \%)$ \\
\hline \multicolumn{6}{|l|}{ Clinical evidence for HIE } \\
\hline$<27 \mathrm{mg} / \mathrm{dL}, n=18$ & $6(33 \%)$ & $5(28 \%)$ & $1(6 \%)$ & $1(6 \%)$ & $5(28 \%)$ \\
\hline $27-45 \mathrm{mg} / \mathrm{dL}, n=38$ & $21(55 \%)$ & $1(3 \%)$ & $5(13 \%)$ & $3(8 \%)$ & $8(21 \%)$ \\
\hline$\geq 46 \mathrm{mg} / \mathrm{dL}, n=104$ & $49(47 \%)$ & $8(8 \%)$ & 19 (18\%) & $6(6 \%)$ & $22(21 \%)$ \\
\hline \multicolumn{6}{|c|}{ No clinical evidence for HIE } \\
\hline$<27 \mathrm{mg} / \mathrm{dL}, n=4$ & $2(50 \%)$ & $2(50 \%)$ & $0(0 \%)$ & $0(0 \%)$ & $0(0 \%)$ \\
\hline $27-45 \mathrm{mg} / \mathrm{dL}, n=0$ & $0(0 \%)$ & $0(0 \%)$ & $0(0 \%)$ & $0(0 \%)$ & $0(0 \%)$ \\
\hline$\geq 46 \mathrm{mg} / \mathrm{dL}, n=15$ & $7(47 \%)$ & $1(7 \%)$ & $0(0 \%)$ & $0(0 \%)$ & $7(47 \%)$ \\
\hline
\end{tabular}

Table 3: Positive and negative predictive values of an MRI pattern of hypoglycemia for clinical hypoglycemia

\begin{tabular}{|c|c|c|c|c|}
\hline & $\begin{array}{l}\text { MRI Pattern } \\
\text { without Clinical } \\
\text { Hypoglycemia }\end{array}$ & $\begin{array}{l}\text { MRI Pattern } \\
\text { with Clinical } \\
\text { Hypoglycemia }\end{array}$ & $\begin{array}{c}\text { Negative } \\
\text { Predictive } \\
\text { Value }\end{array}$ & $\begin{array}{c}\text { Positive } \\
\text { Predictive } \\
\text { Value }\end{array}$ \\
\hline HIE & $5 / 104$ & $25 / 56$ & $76 \%$ & $83 \%$ \\
\hline No HIE & $1 / 15$ & $3 / 4$ & $93 \%$ & $75 \%$ \\
\hline All subjects & $6 / 119$ & $28 / 60$ & $78 \%$ & $82 \%$ \\
\hline
\end{tabular}

$\mathrm{mg} / \mathrm{dL})$. Subjects with NE but without evidence for HIE included 4 of 19 (21\%) with hypoglycemia, all of which was severe.

\section{Radiologic Hypoxic-Ischemic Injury}

As outlined in Table 2, the predominant pattern of injury seen in 179 infants included 85 (47\%) normal studies. When the 19 cases of without evidence for HIE are excluded, the percent distribution of the predominant pattern of injury remains the same as shown in Table 2: $9 \%$ watershed, $14 \%$ basal ganglia, $6 \%$ total, and 23\% focal-multifocal white matter injury. Basal ganglia and total patterns of injury were absent in the group of 19 infants with NE and unconfirmed HIE, whereas 3 (16\%) watershed, 7 (37\%) focalmultifocal, and 9 (47\%) with no injury compose the MR pattern of injury identified in this group.

\section{Radiologic Hypoglycemia}

A radiologic diagnosis of hypoglycemia was made in 34 cases without knowledge of the laboratory glucose values. Of these, 18 (53\%) showed diffusion restriction in the regions of interest. Meanwhile, 13 (38\%) showed changes on conventional T1 and T2 sequences without diffusion restriction. Table 3 correlates the number radiologic hypoglycemic neonates with clinical hypoglycemia $(<46 \mathrm{mg} / \mathrm{dL})$ and the presence or absence of clinical evidence of HIE. Of the 160 infants with confirmed HIE, the MR positive predictive value was $83 \%$ compared with a negative
Table 4: Univariate logistic regression showing the association between clinical hypoglycemia and regional brain injury on MRI, adjusting for umbilical artery $\mathrm{pH}$ and 5-minute Apgar scores

\begin{tabular}{lccr}
\multicolumn{1}{c}{ Injury Region } & $\begin{array}{c}\text { Odds } \\
\text { Ratio }\end{array}$ & $\begin{array}{c}\text { 95\% Confidence } \\
\text { Interval }\end{array}$ & P Value \\
\hline Posterior white matter & 16.29 & $6.29-42.14$ & $<.001$ \\
Posterior gray matter & 4.81 & $1.34-17.28$ & .016 \\
Thalamus, pulvinar & 4.30 & $1.93-9.62$ & $<.001$ \\
Thalamus, anteromedial & 3.21 & $1.23-8.34$ & .017 \\
\hline
\end{tabular}

predictive value of $76 \%$, on the basis of radiologic observation alone. MR imaging findings of hypoglycemia were identified in 5 subjects without clinically documented hypoglycemia, of whom 3 were born at home without glucose testing until after presentation to the hospital at 16, 26, and 39 hours after birth, and 2 had mothers with documented gestational or insulin-dependent diabetes. For glucose values $<46 \mathrm{mg} / \mathrm{dL}, \mathrm{MR}$ imaging only identified 25 of $56(45 \%)$ of the cases. In severe hypoglycemia, $<27 \mathrm{mg} / \mathrm{dL}$, the rate was 13 of $18(72 \%)$ and in extreme cases $(<18 \mathrm{mg} / \mathrm{dL})$ rose to $83 \%(10 / 12)$. Through the use of logistic regression analysis to adjust for 5-minute Apgar scores and umbilical artery $\mathrm{pH}$, clinical hypoglycemia was associated with a 17.6-fold higher odds of identification on MR imaging (95\% confidence interval, 5.754.2; $P<.001$ ). In the 19 cases of NE and unconfirmed HIE, the positive predictive value was $75 \%$ and the negative predictive value was $93 \%$.

Radiologic hypoglycemia was investigated in more detail with univariate logistic analyses performed for each observation; posterior white matter, posterior gray matter, pulvinar, and anteromedial thalamic nuclei (Table 4, Fig 1). A backwards selection model was used to determine which region was most predictive of clinical hypoglycemia, sequentially removing variables with $P$ values $>0.1$. Only the posterior white matter $(P<.001)$ and the pulvinar $(P=.089)$ remained in the model. Through the use of a combination of both posterior white matter and pulvinar injury, 


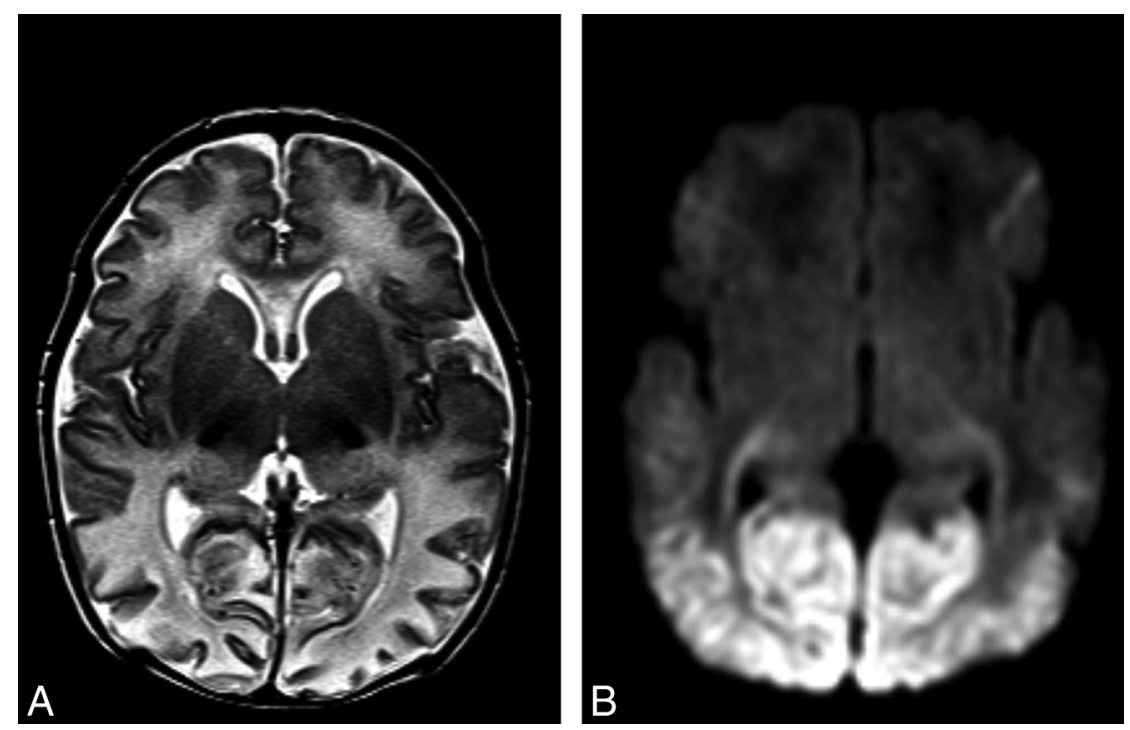

FIG 1. Imaging features of neonatal hypoglycemia superimposed onto HIE. A, T2-weighted MR imaging best shows patchy involvement of the anterior medial and pulvinar nuclei of the thalami. Posterior edema and thinning of the cerebral cortex can also be noted. B, DWI best shows bilateral restricted diffusion involving the optic radiations and bilateral symmetric edema involving both the parieto-occipital white and gray matter.

the positive predictive value was $86 \%$ and the negative predictive value was $77 \%$ for clinical hypoglycemia. Adjusting for umbilical artery $\mathrm{pH}$ and 5-minute Apgar scores, this combined pattern was associated with a 22.58-fold higher odds of clinical hypoglycemia (95\% confidence interval, 6.16-82.86; $P<.001$ ).

\section{DISCUSSION}

Hypoglycemia is an important issue in term neonates with NE and is common in term infants with HIE (34\% in our cohort). Many studies have detailed the radiologic patterns of brain injury in HIE or hypoglycemia, but this large cohort study provides novel information on imaging patterns seen when these 2 conditions coexist. A pattern of posterior white matter and pulvinar injury are most predictive of clinical hypoglycemia superimposed on the predominant pattern of injury seen in HIE. Surprisingly, the watershed pattern of hypoxic-ischemic injury is most frequently seen as associated with severe hypoglycemia, and this pattern coupled with an otherwise normal brain accounts for $>60 \%$ of all cases of hypoglycemia.

Normal MR findings are common in term infants with HIE, but when hypoxic-ischemic brain injury is present, 4 abnormal imaging patterns can be seen: watershed, basal ganglia, total, and focal-multifocal injury. ${ }^{7,8}$ Prolonged partial asphyxia results in a pattern of injury that primarily involves the watershed zones between the major intravascular boundary zones, whereas acute profound asphyxia results in the basal ganglia-predominant pattern of brain injury that involves the basal ganglia, thalami, brain stem, sensorimotor cortex, and corticospinal tracts. ${ }^{1,18,19}$ Severe watershed and basal ganglia-predominant patterns of injury can manifest as total brain injury, occurring when both the cerebral cortex and deep gray nuclei are hypoperfused. ${ }^{18}$ Multifocal predominant pattern of injury describes injury seen in the white matter or cortex. ${ }^{7}$ All predominant patterns of hypoxic-ischemic injury were observed in the current study, with prevalence similar to our previous report. ${ }^{7}$
The first report of brain injury after a case of isolated neonatal hypoglycemia by Spar et $\mathrm{al}^{20}$ in 1994 described a generalized thinning of the cerebral cortex with predominant injury in the parieto-occipital lobes. Few large systematic studies have been performed to characterize the pattern of hypoglycemic brain injury in neonates. One prospective cohort of 35 subjects with hypoglycemia showed posterior white matter abnormalities in $94 \%$ of infants, with other injury of the cortex, white matter hemorrhage, and injury of the basal ganglia, thalamus, and posterior limb of the internal capsule. ${ }^{21}$ A retrospective cohort of 45 neonates with hypoglycemia showed occipital diffusion restriction to be limited to $<7$ days after initial hypoglycemia in term neonates, ${ }^{15}$ and another study of 6 term neonates with hypoglycemia showed restricted diffusion in the parieto-occipital gray and white matter, corpus callosum, and optic radiations. ${ }^{16}$

Animal models suggest increased morbidity and mortality when hypoxic-ischemic injury and neonatal hypoglycemia coexist compared with hypoxic-ischemic injury alone or isolated neonatal hypoglycemia. ${ }^{12,13}$ A cohort of 94 term neonates with encephalopathy showed a $16 \%$ incidence of hypoglycemia, associated with increased odds for corticospinal tract injury. ${ }^{22}$

Higher rates of hypoglycemia have been associated with greater degrees of encephalopathy, ${ }^{4}$ yet in our cohort there was little difference between those with or without hypoglycemia. Even in severe hypoglycemia $(<27 \mathrm{mg} / \mathrm{dL})>60 \%$ of all cases exhibited mild patterns of injury, either watershed or no injury, remarkably similar to the $57 \%$ of term neonates with HIE and normoglycemia. This trend was noticeable in both radiologically positive and clinically positive cases of hypoglycemia. Thus, in severe hypoglycemia, radiologic findings were present in 13 of 18 (72\%) cases; of those, watershed (28\%), focal-multifocal (28\%), and no injury (33\%) composed almost all the cases. One possible explanation of this higher rate of hypoglycemia in neonates with no injury or watershed pattern is that radiologic features of hypo- 
glycemia may be more difficult to appreciate in more severe cases of brain injury. Alternatively, infants with basal ganglia and total injury patterns are sicker and therefore are monitored and managed more closely, which may decrease the rate of hypoglycemia. Of note, neonates with hypoglycemia had smaller birth measurements than their normoglycemic peers. This finding supports the hypothesis that intrauterine growth restriction is a significant risk factor for neonatal hypoglycemia. The findings in our study should underline the importance of close glucose monitoring, not only in the sickest neonates with HIE but even in those who appear to be less acutely affected.

With our large cohort study, we have identified a pattern of brain injury that can help distinguish between hypoglycemic and hypoxic-ischemic injuries. The combination of selective edema in the posterior white matter and pulvinar is most predictive for hypoglycemia in the context of NE and HIE (Fig 1). We have established that this pattern of injury appears to be very specific for hypoglycemia and can be used to indicate an episode of previous hypoglycemia, even in absence of laboratory values or when much of the neonatal history and clinical findings are missing because the infant was born at home or at a remote facility.

Radiologic observations indicating that an infant has had hypoglycemia appear to be robust, but intermittent sampling of glucose values at the discretion of the clinician is not ideal. A closer look at the false-positive and false-negative cases as recorded in this study suggests that the radiologic observations may be even more accurate than stated. Of the $31 \mathrm{MR}$ false-negative cases in HIE documented in Table 2, 6 cases had either a total pattern of injury or massive hemorrhage that precluded recognition of the radiologic pattern of hypoglycemia. Most of the remaining cases were isolated, with either mild decreases in clinical glucose values just $<46 \mathrm{mg} / \mathrm{dL}$ that were immediately corrected, or radiologically normal-appearing brain. In the 160 subjects with HIE, imaging criteria for hypoglycemia were identified in 5 subjects without clinically documented hypoglycemia; of these, however 3 cases were home deliveries, including 2 babies whose mother was diabetic. Similar questions arise in the 19 cases of NE without confirmed HIE, in which there was only 1 false-positive case and 1 false-negative case. It seems therefore reasonable to speculate that the predictive values of radiologic observations of hypoglycemia are even better than documented, but frequent and regular or continuous glucose sampling would be required to ascertain this.

There are limitations to this study. Glucose values were obtained from a retrospective chart review and glucose measurements were obtained at the discretion on the treating physician, resulting in intermittent glucose values and limited information on the duration and severity of neonatal hypoglycemia. The obtained laboratory glucose levels probably underestimate the frequency, severity, and duration of neonatal hypoglycemia in the context of HIE. In addition, clinical measures of HIE such as umbilical artery $\mathrm{pH}$, lactate, and base excess were often missing or not recorded in the chart and limited our ability to adjust for known predictive factors of HIE. As well, neurodevelopmental follow-up was not available in this cohort to assess long-term sequelae associated with this brain injury pattern. Prospective studies correlating this brain injury pattern with neurodevelopmental, including cortical visual, outcome will be important.
Currently, MR imaging is used to identify different patterns of hypoxic-ischemic injury and is a strong predictor of neurodevelopmental outcome. ${ }^{23}$ Our study demonstrates that MR imaging can also reliably identify additional changes of hypoglycemia superimposed on the predominant pattern of injury in HIE and that these changes are more commonly seen with either a normal or watershed injury pattern.

\section{CONCLUSIONS}

In neonates with encephalopathy with clinical evidence for HIE who also had concurrent hypoglycemia, both the predominant pattern of hypoxic-ischemic injury as well as specific imaging features of hypoglycemia could be detected. MR imaging had high positive and negative predictive values for clinical hypoglycemia, both with or without evidence of HIE, indicating that imaging features can be reliably used in the absence of laboratory values to suggest that an infant has had hypoglycemia. Additionally, watershed-predominant hypoxic-ischemic injury was more commonly observed in severe hypoglycemia. Further prospective studies with the use of better measurements of neonatal hypoglycemia are needed to uncover the true relationship between the frequency and duration of neonatal hypoglycemia and hypoxic-ischemic injury and its impact on neurodevelopmental outcome.

\section{ACKNOWLEDGMENTS}

E.W.Y.T. was a Cerebral Palsy International Research Foundation Ethel \& Jack Hausman Clinical Research Scholar. S.P.M. was a Canadian Institutes for Health Research (CIHR) Clinician Scientist and Michael Smith Foundation for Health Research (MSFHR) Scholar.

Disclosures: Darren Wong — RELATED: Support for Travel to Meetings for the Study or Other Purposes: travel costs, Comments: Dr Steven Miller (a co-author) sponsored my trip to the Pediatric Academic Societies meeting in Boston, Massachusetts, to present part of these findings in a poster symposium session. Steven Miller-UNRELATED: Expert Testimony: Canadian Medical Protective Association, Comments: Review of legal files related to suspected instances of birth asphyxia. Emily W.Y. TamRELATED: Grant: Cerebral Palsy International Research Foundation,* Comments: Scholars Award; UNRELATED: Grants/Grants Pending: NIH, Gerber Foundation. ( ${ }^{*}$ Money paid to institution.)

\section{REFERENCES}

1. Volpe JJ. Neurology of the Newborn. Philadelphia: WB Saunders Company; 2008

2. Phelan JP, Martin GI, Korst LM. Birth asphyxia and cerebral palsy. Clin Perinatol 2005;32:61-76

3. Barkovich AJ, Miller SP, Bartha A, et al. MR imaging, MR spectroscopy, and diffusion tensor imaging of sequential studies in neonates with encephalopathy. AJNR Am J Neuroradiol 2006;27:533-47

4. Basu P, Som S, Choudhuri N, et al. Contribution of the blood glucose level in perinatal asphyxia. Eur J Pediatr 2009;168:833-38

5. Chau V, Poskitt KJ, Miller SP. Advanced neuroimaging techniques for the term newborn with encephalopathy. Pediatr Neurol 2009;40: 181-88

6. Miller SP. Neonatal encephalopathy. In: Johnson RT, ed. Current Therapy in Neurologic Disease. 7th ed. Philadelphia: Mosby; 2006: 89-91

7. Chau V, Poskitt KJ, Sargent MA, et al. Comparison of computer tomography and magnetic resonance imaging scans on the third day of life in term newborns with neonatal encephalopathy. Pediatrics 2009; 123:319-26

8. Barkovich AJ, Ali FA, Rowley HA, et al. Imaging patterns of neonatal hypoglycemia. AJNR Am J Neuroradiol 1998;19:523-28 
9. Montassir H, Maegaki Y, Ohno K, et al. Long term prognosis of symptomatic occipital lobe epilepsy secondary to neonatal hypoglycemia. Epilepsy Res 2010;88:93-99

10. Udani V, Munot P, Ursekar M, et al. Neonatal hypoglycemic brain injury: a common cause of infantile onset remote symptomatic epilepsy. Indian Pediatr 2009;46:127-32

11. Lucas A, Morley R, Cole TJ. Adverse neurodevelopmental outcome of moderate neonatal hypoglycaemia. BMJ 1988;297:1304-08

12. Vannucci RC, Vannucci SJ. Cerebral carbohydrate metabolism during hypoglycemia and anoxia in newborn rats. Ann Neurol 1978;4:73-79

13. Young RS, Cowan BE, Petroff $\mathrm{OA}$, et al. In vivo $31 \mathrm{P}$ and in vitro $1 \mathrm{H}$ nuclear magnetic resonance study of hypoglycemia during neonatal seizure. Ann Neurol 1987;22:622-28

14. Nadeem M, Murray DM, Boylan GB, et al. Early blood glucose profile and neurodevelopmental outcome at two years in neonatal hypoxic-ischaemic encephalopathy. BMC Pediatr 2011;11:10

15. Tam EW, Widjaja E, Blaser SI, et al. Occipital lobe injury and cortical visual outcomes after neonatal hypoglycemia. Pediatrics 2008;122: $507-12$

16. Filan PM, Inder TE, Cameron FJ, et al. Neonatal hypoglycemia and occipital cerebral injury. J Pediatr 2006;148:552-55
17. Barkovich AJ, Hajnal BL, Vigneron D, et al. Prediction of neuromotor outcome in perinatal asphyxia: evaluation of MR scoring systems. AJNR Am J Neuroradiol 1998;19:143-49

18. Barkovich AJ. Injury in the term infant. In: Pediatric Neuroimaging. Philadelphia: Lippincott Williams \& Wilkins; 2005:224-40

19. Roland EH, Poskitt K, Rodriguez E, et al. Perinatal hypoxic-ischemic thalamic injury: clinical features and neuroimaging. Ann Neurol 1998;44:161-66

20. Spar JA, Lewine JD, Orrison WW Jr. Neonatal hypoglycemia: CT and MR findings. AJNR Am J Neuroradiol 1994;15:1477-78

21. Burns CM, Rutherford MA, Boardman JP, et al. Patterns of cerebral injury and neurodevelopmental outcomes after symptomatic neonatal hypoglycemia. Pediatrics 2008;122:65-74

22. Tam EW, Haeusslein LA, Bonifacio SL, et al. Hypoglycemia is associated with increased risk for brain injury and adverse neurodevelopmental outcome in neonates at risk for encephalopathy. J Pediatr 2012;161:88-93

23. Miller SP, Latal B, Clark H, et al. Clinical signs predict 30-month neurodevelopmental outcome after neonatal encephalopathy. Am J Obstet Gynecol 2004;190:93-99 